\title{
A Multicenter Retrospective Analysis of the Long-Term Efficacy and Safety of a Novel Posterior Sacroiliac Fusion Device
}

\author{
Dawood Sayed (ID) \\ Kevin Balter ${ }^{2,3}$ \\ Stephen Pyles ${ }^{4}$ \\ Christopher M Lam (D) \\ 'Department of Anesthesiology and Pain \\ Medicine, University of Kansas Medical \\ Center, Kansas City, KS, USA; ${ }^{2}$ Midwest \\ Pain Clinics, Omaha, NE, USA; \\ ${ }^{3}$ Department of Internal Medicine, \\ Creighton University School of Medicine, \\ Omaha, NE, USA; ${ }^{4}$ Pain Treatment \\ Centers, Ocala, FL, USA
}

Correspondence: Christopher M Lam Department of Anesthesiology and Pain Medicine, University of Kansas Medical Center, 390I Rainbow Boulevard, Kansas City, Kansas City, 66160, USA

Tel + I 9139459810

$\mathrm{Fax}+1$ 913-588-3365

Email clam2@kumc.edu
Background: Sacroiliac joint (SIJ) pathology is a cause of low back pain that may be difficult to diagnose and challenging to treat. Open and minimally invasive (MI) lateral approach fusions have been used to treat sacroiliitis over the past two decades. A novel MI posterior approach SIJ fusion technique utilizes a posteriorly placed transfixing device with single point S1/S2 level or mid-segment SIJ fixation (LinQ procedure). Current efficacy and safety data for this novel procedure are lacking.

Objective: To review multicenter retrospective 12 months or greater outcomes data in patients receiving the LinQ procedure, with sub-analysis of patients with prior lumbar fusions.

Methods: Patients with sacroiliitis refractory to conservative care with short-term benefit from diagnostic local anesthetic SIJ injections receiving MI posterior approach SIJ fusion with allograft were included from different centers including both academic and private practice. Numeric rating scale (NRS) scores at baseline (pre-procedural) and most recent follow-up were reviewed across three institutions.

Results: Of 110 patients who received MI SIJ fusion, 50 patients had sufficient data for evaluation of outcomes at least 12 months post-implant. The average time out from implant at follow-up was 612.2 days for all unique patients. The average NRS was 6.98 pre-fusion and 3.06 at last follow-up. Twenty-four patients had prior lumbar surgery of which 17 had prior lumbar fusions. Average NRS for this subset was 6.85 at baseline and 2.86 at last follow-up with an average follow-up of 613.2 days out from implant. No major adverse events or complications were associated with any of the 50 implants.

Conclusion: Real-world evidence suggests that MI posterior SIJ fusion with the LinQ procedure is a viable approach for medically refractory sacroiliitis management with long-term efficacy and safety. Further prospective studies are needed to fully evaluate this technique.

Keywords: sacroiliitis, sacroiliac joint, minimally invasive intervention, allograft, low back pain, buttock pain

\section{Introduction}

Low back pain presents a large burden to the US healthcare system. It has been reported that roughly $\$ 86$ billion dollars were spent for the investigation and treatment of this condition in 2008. ${ }^{1}$ The sacroiliac joint (SIJ) is a diarthrodial joint that, when dysfunctional, is a common cause of low back pain. ${ }^{2}$

Studies suggest that sacroiliitis may account for $10-26 \%$ of all cases of suspected low back pain. ${ }^{3}$ The accuracy of provocative measures, imaging, and history of the pain pattern associated with this condition for diagnosis is limited. ${ }^{4,5}$ There is 
moderate evidence for the specificity and accuracy of the diagnostic SIJ injection. ${ }^{3}$ Equally challenging is the management of this condition with medications, physical therapy, bracing, SIJ intraarticular steroid injections, and radiofrequency ablative techniques, many of which do not always offer long-lasting relief. Many of the aforementioned approaches require further studies in order to validate their efficacy in a larger population. ${ }^{1,6-8}$

Historically, open sacroiliac fusions with hardware placement have been utilized to address this condition. Initial methodologies included bilateral fusion via posterolateral approach. In a study by Schutz and Grob, 14 of 17 patients $(82 \%)$ who received this surgery reported persistent marked or severe pain at follow up. ${ }^{9}$ Furthermore, there were significant risks associated with this approach including mean operative time of 121 minutes, estimated procedural blood loss of $793 \mathrm{~mL}$, and mean length of hospitalization of 25.2 days. ${ }^{4}$ Further advances in open approaches included utilization of devices such as a "T-Plate" which resulted in an $85 \%$ fusion rate, $20 \%$ complication rate, and $60 \%$ patient satisfaction. ${ }^{10}$ Despite advances in surgical technique and implant technology, open approach SIJ fusion is associated with high overall rates of complications ranging from $13 \%$ to $21 \%{ }^{4}$

Minimally invasive (MI) fusion for SIJ disease has been explored since 1998, historically utilizing a lateral incisional approach, with reported improvement lasting up to 4.5 years post-operatively with consequent concomitant decreased opioid consumption. ${ }^{4}$ Studies comparing open to MI fusion have shown that MI fusion was associated with greater Visual Analog Scale (VAS) reduction at 24 months follow up, shorter operative time, less estimated blood loss, and shorter hospital length of stay. ${ }^{11,12}$ Though the initial cost of the procedure exceeds that of conservative medical managements, studies have implicated that the cost of SIJ fusion is offset within 5 years post-fusion compared with continued medical management with cost neutrality achieved at 6 years. ${ }^{13}$

Patients with a prior lower segment lumbar fusion may present with adjacent segment disease (ASD) affecting the SIJ. Overall reported incidence of ASD in patients with lumbar fusion was as high as $30 \% .{ }^{14}$ Other studies have indicated that $75 \%$ of patients 5 years out from a lumbar fusion will have radiologic findings of degenerative changes in the sacroiliac joint. ${ }^{6}$ Risk factors for this include fusion length, sagittal malalignment, facet injury, and advanced age. ${ }^{14}$ Furthermore, studies have reported that patients with lumbar pathology may not benefit as much with SIJ management including fusion compared with patients without lumbar disease or surgery. ${ }^{15}$

Posterior approach sacroiliac fusion is a new minimally invasive technique to address sacroiliitis. The LinQ posterior sacroiliac fusion system is an FDA approved device used for the transfixation of patients with sacroiliac dysfunction. The system utilizes a patented cortical allograft as well as a patented drill-less method for posterior sacroiliac fusion (Figures 1 and 2). However, there are limited reported data on this procedure. Here, we present initial multicenter retrospective data on a minimally invasive posterior approach SIJ fusion technique utilizing a posteriorly placed allograft with single point fixation at the S1/S2 or mid segment of the sacroiliac joint, with subanalysis in patients with a history of prior lumbar fusion.

\section{Materials and Methods}

A multicentered, retrospective chart review was performed on the charts of all eligible patients from October 1, 2018 to April 1, 2020. Three providers at 3 different institutions contributed de-identified retrospective data and independently evaluated patient records. One center was a tertiary academic medical center and the other two sites were community based private practice settings. An IRB waiver was granted by Sterling IRB to the primary investigator who provided oversight for the study (IRB\# 8073) given the retrospective nature of the study without need to further contact the involved patients. All patient data collected were de-identified to provide patient data confidentiality and compliance with the Declaration of Helsinki. The protocol and IRB were each approved by the local governing entities of each involved institution.

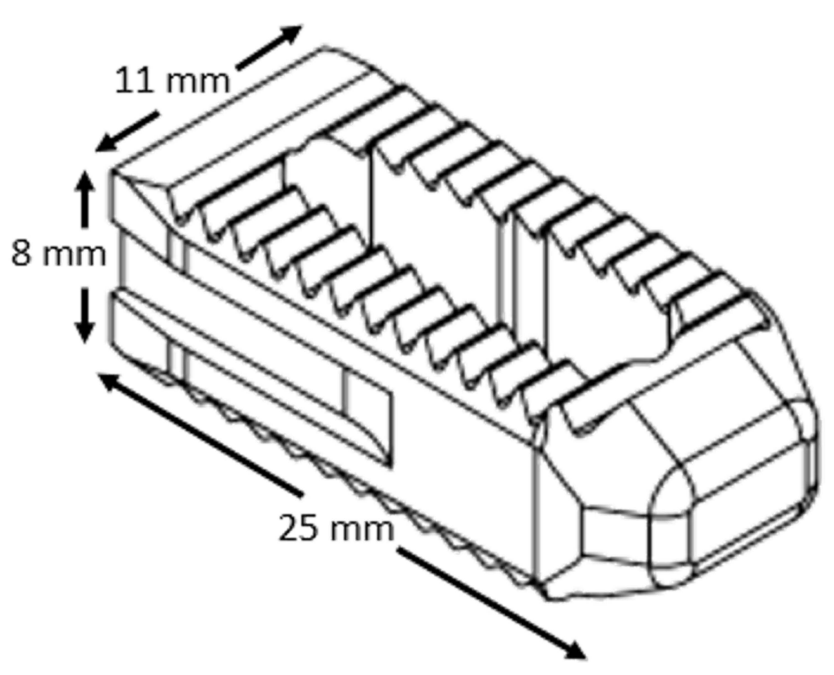

Figure I Schematic of cortical allograft. 
1. Guide Pin Storage Tube

2. Implant Inserter

3. Joint Decorticator

4. Outside Dilator/Working Channel

5. Inside Dilator

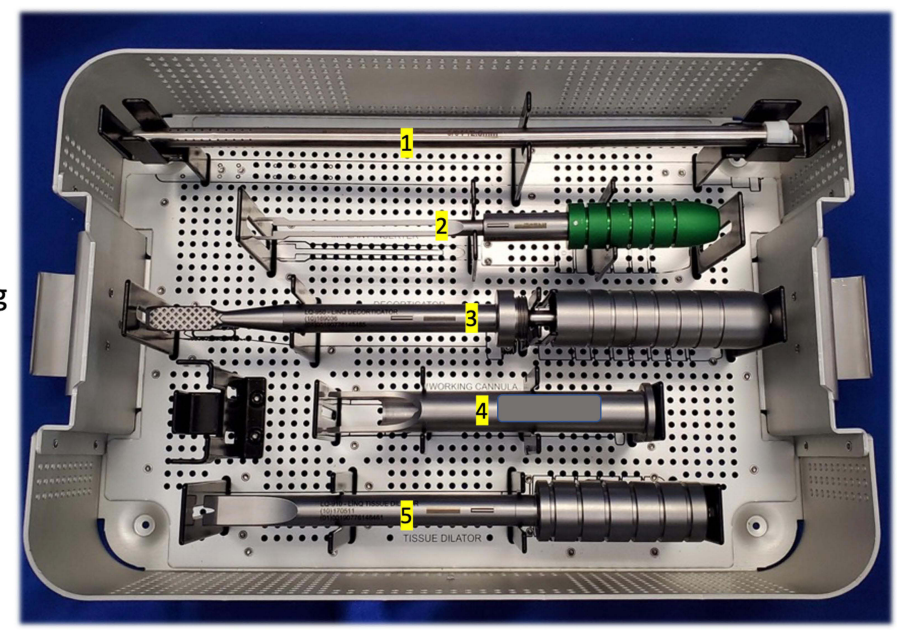

Figure 2 Instrument set for MI percutaneous SIJ fusion.

Patients selected for this review included those who had a documented history and physical exam findings of sacroiliitis, failed conservative management, undergone at least one satisfactory diagnostic local anesthetic SIJ injection, and underwent MI posterior approach SIJ fusion with the LinQ Fusion Device (PainTeq, Tampa, FL). Physical exam findings must have included 3 out of 5 possible provocative maneuvers of sacroiliitis: SIJ distraction, thigh thrust, FABER test, compression test, and Gaenslen's maneuver. Patients excluded from the study include those that had not reached the desired 12-month assessment time mark at the time of this retrospective review to accurately detail longterm efficacy of their response to therapy.

The SIJ injection was performed with the patient in prone positioning under fluoroscopic guidance to line up the medial and lateral borders of the joint. Using sterile technique with adequate sterilization of patient skin, local anesthetic was applied along the trajectory of proposed needle path and a spinal needle was advanced until it entered the joint space. Correct placement was confirmed with injection of contrast under fluoroscopic imaging before local anesthetic was injected. All providers performed diagnostic local anesthetic SIJ injections with a similar approach, and each independently interpreted the results of the injections. If greater than $50 \%$ relief was achieved, patients were deemed candidates for MI posterior approach SIJ fusion.

The MI posterior approach SIJ fusion was performed with guidance of fluoroscopy on patients in prone positioning as noted previously by Lee et al. ${ }^{16}$ The medial and lateral borders of the SIJ were identified and lined up to allow for spinal needle placement into the joint at the S1/S2 segment to apply local anesthetic to the skin, underlying tissues, and into the SIJ joint.
A 2-3 cm incision was made along the skin and a Steinmann pin was advanced until it had entered the middle $1 / 3$ of the sacroiliac joint (Figures 3 and 4A). Correct placement was confirmed with fluoroscopy prior to joint dilation. Following sequential dilation utilizing an inside dilator, a specialized outside dilator was placed across the joint to allow for decortication across the sacrum and ilium posteriorly with a joint decorticator instrument and mallet until the instrument was fully seated (Figures 3, 4B and C). Once seated, the decorticator was removed utilizing a reverse slap hammer. Subsequently, cortical allograft transfixing device packed with demineralized bone matrix (DBM) putty was prepared and placed across the dilated sacroiliac joint until fully seated to stabilize and promote fusion (Figures 3 and 4D). Once placement was confirmed, the dilating device and all other instruments were removed before radiographic confirmation of correct placement (Figures 3, 4E and F). The wound was then irrigated, hemostasis obtained, and incision closed in a staged manner. Pre-fusion baseline numeric rating scale (NRS) score, NRS at most recent follow up, and procedural complication events were obtained from the respective patient charts. Available patient demographics were also collected including age, history of prior lumbar surgery, and lumbar fusion level. Descriptive statistics were calculated and are reported below.

\section{Results}

In total, 110 charts were reviewed of patients who had the MI posterior approach SIJ fusion performed. A chart analysis revealed 50 of the 110 patients had pre-fusion and postfusion NRS scores and were at least 12 months out from implant. Patients excluded were not 12 months out from 


\section{Implant Procedure}

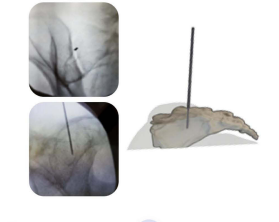

-

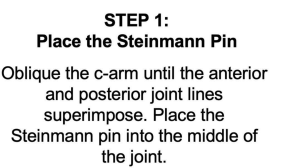

STEP 2: Place the Dilators

Slide the Inside Dilator into the Outside Dilator. Advance the Dilators down the Steinmann pin until it is fully seated in the SI joint. Remove the Inside Dilator and Steinmann pin.

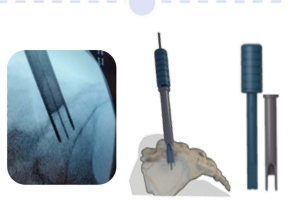

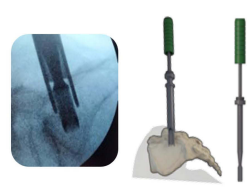

STEP 3

Decorticate the Joint

Place the Joint Decorticator into the Outside Dilator. Lightly tap with a mallet until the Joint Decorticator is fully seated. Remove the Decorticator by unscrewing the handle and using it as a reverse
slap hammer.

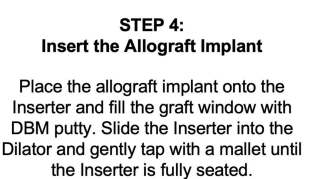

the Inserter is fully seated.
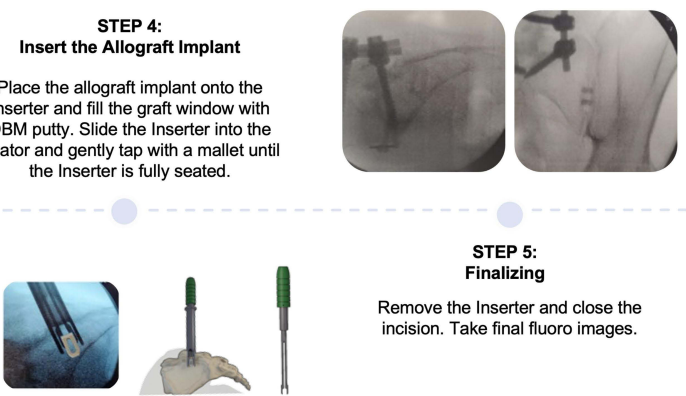

\section{STEP 5:
Finalizing \\ Remove the Inserter and close the incision. Take final fluoro images.}

Figure 3 Schematic for procedural steps of implantation.
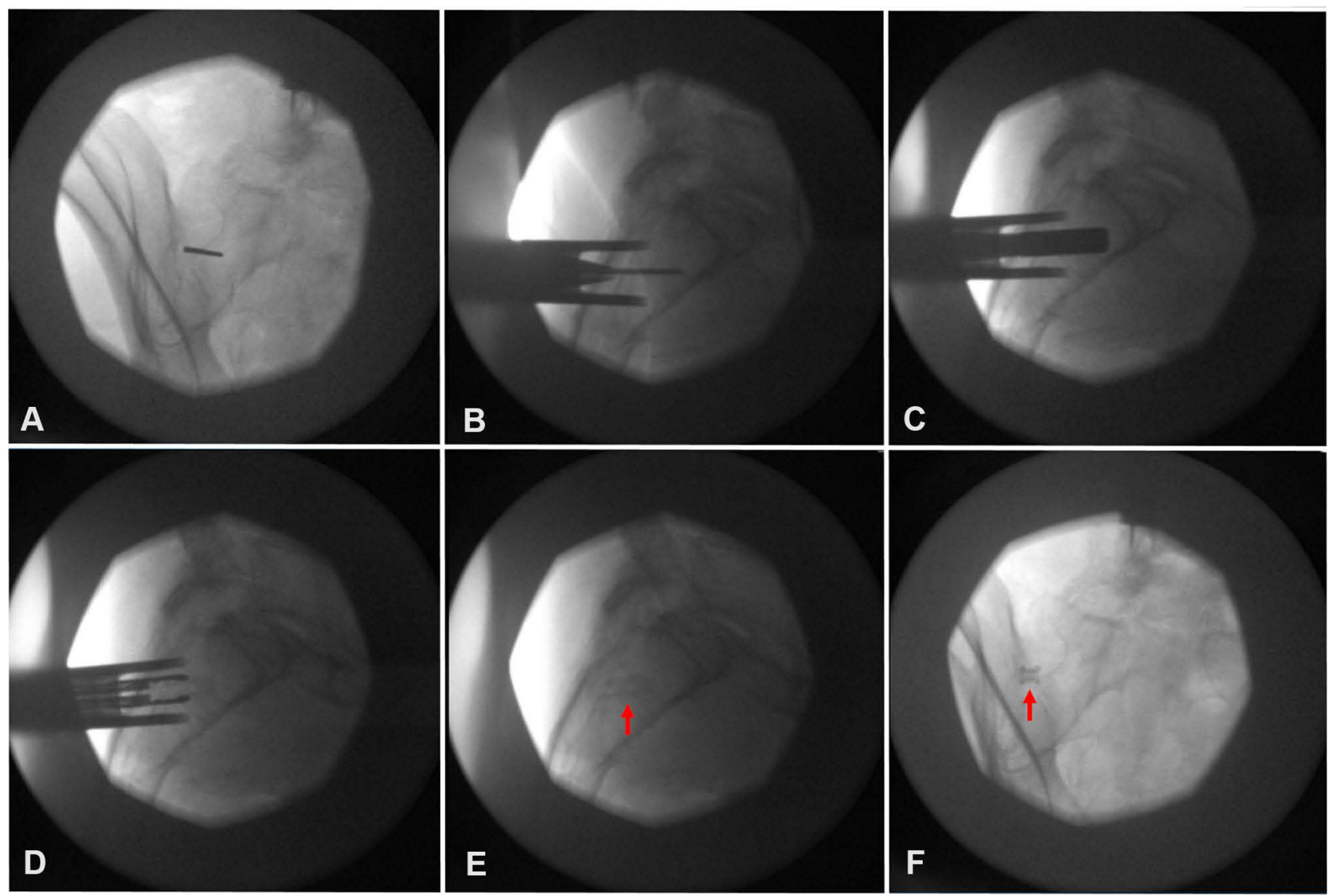

Figure 4 Intraprocedural Imaging. (A) Oblique view for Steinmann pin placement. (B) Dilator placement over Steinmann pin into SIJ assessed by lateral fluoroscopic view. (C) SIJ decortication in lateral fluoroscopic view. (D) Graft deployment device insertion lateral fluoroscopic view. (E) Deployed graft in lateral fluoroscopic view, indicated by arrow marker. (F) Final anterior posterior view of deployed graft, indicated by arrow marker.

implant or had not presented for their 12-month evaluation at the time of this retrospective chart review so that we only reported on long-term efficacy of the procedure.
Approximately $48.0 \%$ of the cohort were males $(\mathrm{N}=24)$ while $52.0 \%$ were females $(\mathrm{N}=26)$. Analysis was performed on this group of 50 patients. The average age of the patients in 
Table I Patient Demographics

\begin{tabular}{|l|r|}
\hline & Mean 士 SD or $\mathbf{n}(\%)$ \\
\hline Male & $24(48.0 \%)$ \\
Female & $26(52.0 \%)$ \\
Averagnostic Injection Relief & $90.6 \pm 15.9$ \\
Average length of follow up (Days) & $68.0 \pm 12$ \\
Baseline NRS Pain & $612.2 \pm 116.1$ \\
No History of Lumbar Surgery & $6.98 \pm 2.61$ \\
History of Lumbar Surgery & $26(52.0 \%)$ \\
\hline
\end{tabular}

this cohort was 68.0 years old with an average length of follow up of 612.2 days (Table 1). The overall average pre SIJ fusion NRS was 6.98 (95\% CI [6.26, 7.70]) with percent relief from diagnostic injection noted to be $90.6 \%$. The overall average NRS at last follow up was 3.06 (95\% CI [2.35, 3.77]) with an average overall percent relief of $66.5 \%$ (Figures 5 and 6). Seven patients in this cohort had satisfactory relief of SIJ fusion and decided to obtain contralateral SIJ fusion by similar approach (14.0\%).

The NRS at baseline and most recent follow up in patients with no history of lumbar surgery $(\mathrm{N}=26)$ were 7.74 (95\% CI [7.08, 8.40]) and 3.75 (95\% CI [2.69, 4.81]) (Figure 5). Meanwhile, the NRS at baseline and most recent follow up in patients with history of any lumbar surgery $(\mathrm{N}=24)$ was $6.32(95 \% \mathrm{CI}[5.12,7.52])$ and 2.43 (95\% CI [1.52, 3.35]) (Figure 3). Of patients with prior lumbar surgery, 17 had prior lumbar fusions, of which 9 had fusions involving L5-S1. The average age of patients with history of prior lumbar fusion was 63.3 years old. The average pre-fusion NRS was 6.85 (95\% CI [5.48, 8.22]) and the average relief from the diagnostic injection was 93.6\%. Average NRS at last follow up was 2.86 (95\% CI $[1.72,3.99])$ with $66.8 \%$ patient-reported relief in those with history of lumbar fusion. When compared with patients with any lumbar surgery or lumbar fusion, the NRS at last follow up was statistically significantly higher $(p=0.03)$ in patients without history of prior lumbar surgery. Further, the patient-reported percent relief at most recent follow up for patients without history of surgery receiving an SIJ fusion device was $59.6 \%$ while those with history of any lumbar surgery reported their relief at $71.9 \%$.

There were no serious adverse events or major complications associated with any of the 50 implants, defined as surgical site infection, perioperative hemorrhage, or death. However, there was an episode of device migration that was observed in one patient requiring placement of a second allograft to further stabilize the joint (2.0\%). This patient subsequently reported $100 \%$ relief with an NRS of 0 at the most recent follow up.

\section{Discussion}

This multi-institutional review demonstrates that patients with persistent sacroiliitis despite usual medical management

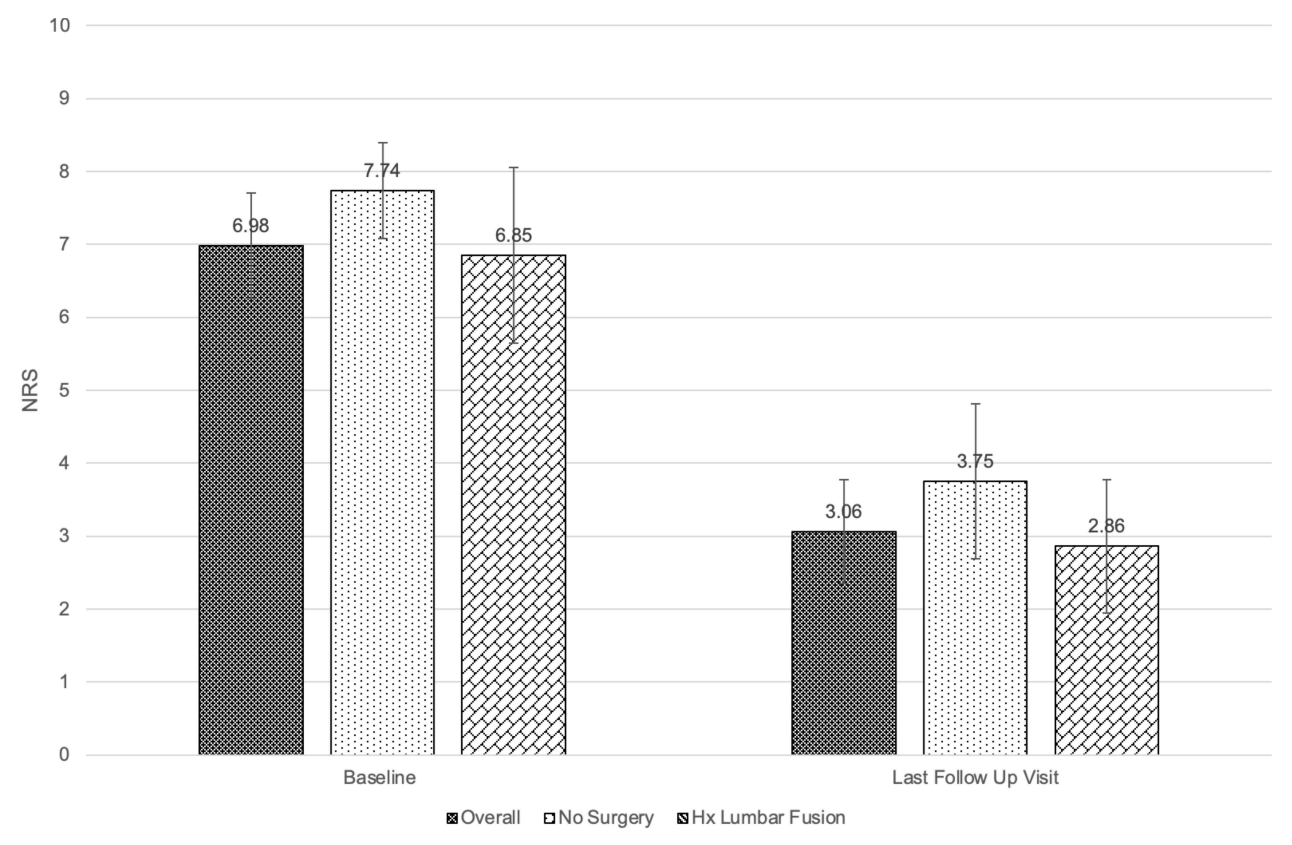

Figure 5 Baseline and most recent follow up patient-reported numeric rating scale score. 


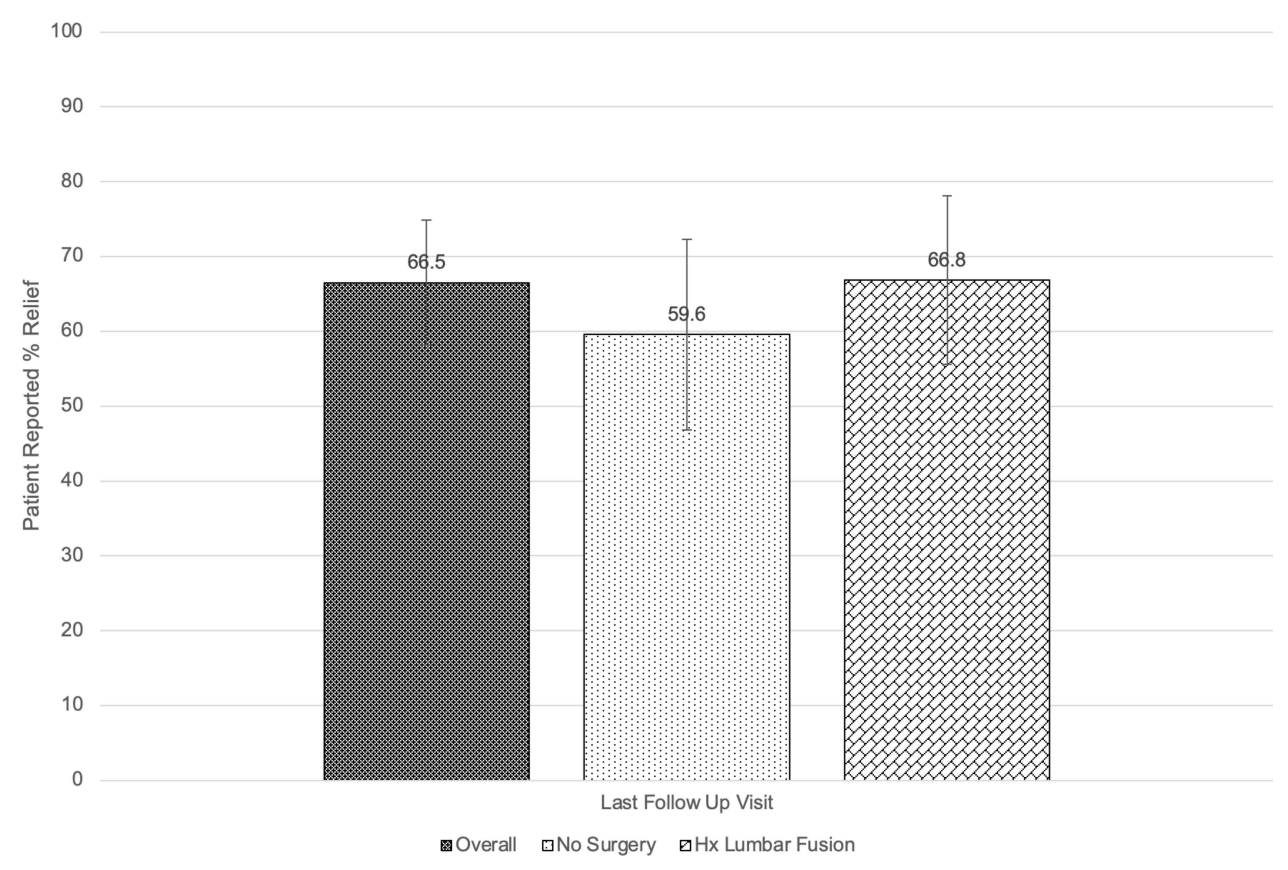

Figure 6 Patient-reported percent relief after posterior percutaneous SIJ fusion at most recent follow up.

benefitted with improved overall pain control after receiving MI posterior approach SIJ fusion. We observed a sustained large reduction of patient NRS, of at least 3, at most recent follow up compared with baseline. Aside from NRS reduction, patients reported an average of $66.5 \%$ improvement of their symptoms without need for any repeat SIJ intervention, save for one episode of device migration requiring a second implant that resulted in sustained relief without further intervention thereafter. These findings are similar to those reported in a prospective randomized control study by Polly et al. evaluating 2-year outcomes in patients with SIJ dysfunction treated with minimally invasive lateral approach SIJ fusion utilizing titanium rods compared with non-surgical management. At 2 years, patients undergoing SIJ fusion had a mean improvement of 55.4 by VAS compared with those with non-surgical management, resulting in $83.1 \%$ of patients with VAS improvement. ${ }^{17}$ A literature review by Martin et al. including all studies on sacroiliac joint fusion surgeries with at least 12 month follow up revealed a mean decrease of 48.1 VAS and ODI decrease of 21.8. ${ }^{18}$ A recent publication by Deer et al. detailed a retrospective chart review of patients with a history of previously implanted pain devices and therapies receiving a posterior MI SIJ fusion device as a salvage therapy resulted in a mean patient reported pain relief of $67.6 \% .{ }^{19}$ Similar to these studies, our cohort reported an overall patient reported pain relief of
$66.5 \%$ and NRS reduction of at least 3 , over a year out from initial implant.

Notably, patients in this review reported relief that was sustained on average 612 days after implant. Each provider has similar post-procedural activity restrictions which allowed for full weight bearing and minimal activity restrictions post-operatively. Variations in patient's habitus, activity level, and other comorbid conditions may affect the rate and fidelity of device integration into the joint resulting in the discrepancies in reported relief as 11 patients $(22 \%)$ reported $<50 \%$ relief at most recent follow up. Of this group, five patients report no relief after implant even over 12 months out. This may indicate a potential failure rate of $10 \%$ and a potential success rate of $78 \%$.

Patients with prior lumbar fusion were sub-analyzed in this cohort, as it is hypothesized that these patients may be at higher risk of developing SIJ pain. These patients had a slightly lower baseline NRS compared with the patients without history of lumbar fusion $(6.85$ vs $7.74, \mathrm{p}=$ $0.0001)$. This difference was sustained at the most recent follow up as patients with prior lumbar fusions reported a lower NRS (2.86 vs $3.75, p=0.03$ ). Though statistically significant, this difference is likely not clinically significant. Given the limited scope of this review and the observational retrospective nature of the study, the details 
recorded were not sufficient to characterize or indicate risk factors for device failure or success.

Estimated blood loss data were incomplete and could not be analyzed, although we can report that no patients required intraoperative blood products or transfusion. All procedures were performed on a same day, outpatient basis with no extended stay in recovery. The procedure was performed percutaneously with one access site. None of the patients suffered any immediate complications or serious adverse outcomes from this procedure. In a subset of patients with bilateral sacroiliitis with significant improvement from initial single site fusion, a second contralateral fusion was performed. Long-term follow up of these patients yielded one significant device migration presenting as recurrent SIJ pain made worse with provocative maneuvers. To address this, the first device was not removed and a second implant was used to further stabilize the joint that resulted in sustained relief in that particular patient. Radiographs of the device after implantation were not routinely obtained thus we were not able to measure or record any slight or small migrations to fully evaluate rate of fusion. However, non-fusion may explain the pain scores of some patients who reported suboptimal relief. Otherwise, no incidences of localized infection, systemic infection, bleeding, transfusion of blood products, neurologic injury, or iatrogenic fractures of the sacrum or surrounding bone structures were reported with long-term follow up. This may be partially explained by the small sample size included in this study not being large enough to capture the true rate of adverse events associated with this procedure. Though not a major adverse event, device migration is a complication of the procedure with the occurrence of $2.0 \%$ in our 50 patient cohort.

This study has several limitations. It is a retrospective observational study to evaluate the rate of complications associated with device utilization and patient-reported outcomes. Due to the timing of initial chart review, many of the patients excluded were not 12 months out from their initial implant or have not presented for their 12 month follow up. To avoid further bias and confounding factors, patients with insufficient data at our desired end point of 12 months were excluded. This resulted in a smaller sample size. Being a relatively new procedure and the need for sufficient follow up to truly evaluate therapy efficacy, time mark of at least 12 months post-implant was utilized as our primary end point. Because of this, many patients were subsequently ineligible to be included in this review.
However, it is our goal to provide observational longterm results for this new therapy to provide guidance until prospective studies are complete to further delineate the efficacy of this therapy.

As a result, this is not a comparative study to delineate if this is superior to other means of management. A standard protocol is needed to evaluate device fixation and arthrodesis to fully gauge the fidelity of the device remaining fixed in the joint. A prospective multicenter study on this surgical technique is currently in progress. Further, the data reviewed are not sufficient to discuss risk factors for device failure or success. This was not a hypothesis-driven study, so findings are observational only and no intention-to-treat analysis was performed. Though significant, the results observed here may have multiple confounding factors including recall bias, response bias, and interviewer bias as patients have failed traditional therapies prior to participating in the SIJ fusion. Further structured multicenter prospective studies are needed to fully evaluate this technique for management of sacroiliitis.

\section{Conclusion}

The data presented here indicate that this approach may be a viable means for treatment of sacroiliitis with possible long-term efficacy, illustrating the need for further welldesigned controlled studies to verify these findings. Although further prospective studies are clearly warranted, this dataset serves as a promising preamble for future studies.

\section{Disclosure}

DS reports funded research with PainTeq, Medical Advisory Board of PainTeq, and minority options in PainTeq. He also reports personal fees from Abbott, Medtronic, Nevro, and Flowonix; personal fees and options from Vertos, outside the submitted work. SP reports minority options in PainTeq. The authors report no other conflicts of interest in this work.

\section{References}

1. Bina RW, Hurlbert RJ. Sacroiliac fusion: another "magic bullet" destined for disrepute. Neurosurg Clin N Am. 2017;28(3):313-320. doi:10.1016/j.nec.2017.02.001

2. Amirdelfan K, McRoberts P, Deer TR. The differential diagnosis of low back pain: a primer on the evolving paradigm. Neuromodulation. 2014;17(Suppl 2):11-17. doi:10.1111/ner.12173

3. Hansen HC, Brown AM, Cohen SP, Swicegood JR, Colson JD, Manchikanti L. Sacroiliac joint interventions: a systematic review. Pain Physician. 2007;10(1):165-184. doi:10.36076/ppj.2007/10/165 
4. Ou-Yang DC, York PJ, Kleck CJ, Patel VV. Diagnosis and management of sacroiliac joint dysfunction. J Bone Joint Surg Am. 2017;99 (23):2027-2036. doi:10.2106/JBJS.17.00245

5. van der Wurff P, Hagmeijer RH, Meyne W. Clinical tests of the sacroiliac joint. A systematic methodological review. Part 1: reliability. Man Ther. 2000;5(1):30-36. doi:10.1054/math.1999.0228

6. Rashbaum RF, Ohnmeiss DD, Lindley EM, et al. Sacroiliac joint pain and its treatment. Clin Spine Surg. 2016;29(2):42-48. doi:10.1097/ BSD.0000000000000359

7. Al-Subahi M, Alayat M, Alshehri MA, et al. The effectiveness of physiotherapy interventions for sacroiliac joint dysfunction: a systematic review. J Phys Ther Sci. 2017;29(9):1689-1694. doi:10.1589/jpts.29.1689

8. Facchini G, Spinnato P, Guglielmi G, et al. A comprehensive review of pulsed radiofrequency in the treatment of pain associated with different spinal conditions. Br J Radiol. 2017;90(1073):20150406. doi:10.1259/bjr.20150406

9. Schutz U, Grob D. Poor outcome following bilateral sacroiliac joint fusion for degenerative sacroiliac joint syndrome. Acta Orthop Belg. 2006;72(3):296-308.

10. Buchowski JM, Kebaish KM, Sinkov V, et al. Functional and radiographic outcome of sacroiliac arthrodesis for the disorders of the sacroiliac joint. Spine J. 2005;5(5):520-528; discussion 529. doi:10.1016/j.spinee.2005.02.022

11. Smith AG, Capobianco R, Cher D, et al. Open versus minimally invasive sacroiliac joint fusion: a multi-center comparison of perioperative measures and clinical outcomes. Ann Surg Innov Res. 2013;7 (1):14. doi:10.1186/1750-1164-7-14

12. Ledonio CG, Polly DW Jr, Swiontkowski MF. Minimally invasive versus open sacroiliac joint fusion: are they similarly safe and effective? Clin Orthop Relat Res. 2014;472(6):1831-1838. doi:10.1007/s11999-014-3499-8
13. Ackerman SJ, Knight T, Schneider K, et al. Comparison of the costs of nonoperative care to minimally invasive surgery for sacroiliac joint disruption and degenerative sacroiliitis in a United States commercial payer population: potential economic implications of a new minimally invasive technology. Clinicoecon Outcomes Res. 2014;6:283-296. doi:10.2147/CEOR.S63757

14. Epstein NE. Adjacent level disease following lumbar spine surgery: a review. Surg Neurol Int. 2015;6(Suppl 24):S591-9. doi:10.4103/ 2152-7806.170432

15. Rudolf L. MIS fusion of the SI joint: does prior lumbar spinal fusion affect patient outcomes? Open Orthop J. 2013;7(1):163-168. doi: $10.2174 / 1874325001307010163$

16. Lee DW, Patterson DG, Sayed D. Review of current evidence for minimally invasive posterior sacroiliac joint fusion. Int J Spine Surg. 2021;15(3):514-524. doi:10.14444/8073

17. Polly DW, Swofford J, Whang PG, et al. Two-year outcomes from a randomized controlled trial of minimally invasive sacroiliac joint fusion vs. non-surgical management for sacroiliac joint dysfunction. Int J Spine Surg. 2016;10:28. doi:10.14444/3028

18. Martin CT, Haase L, Lender PA, et al. Minimally invasive sacroiliac joint fusion: the current evidence. Int J Spine Surg. 2020;14(Suppl 1):20-29. doi:10.14444/6072

19. Deer TR, Rupp A, Budwany R, et al. Pain relief salvage with novel minimally invasive posterior sacroiliac joint fusion device in patients with previously implanted pain devices and therapies. J Pain Res. 2021;14:2709-2715. doi:10.2147/JPR.S325059
Journal of Pain Research

\section{Publish your work in this journal}

The Journal of Pain Research is an international, peer reviewed, open access, online journal that welcomes laboratory and clinical findings in the fields of pain research and the prevention and management of pain. Original research, reviews, symposium reports, hypothesis formation and commentaries are all considered for publication. The manuscript

\section{Dovepress}

management system is completely online and includes a very quick and fair peer-review system, which is all easy to use. Visit http:// www.dovepress.com/testimonials.php to read real quotes from published authors. 